Ecology

Elsevier Editorial System(tm) for Fungal

Manuscript Draft

Manuscript Number: FUNECO-D-17-00277R1

Title: DO YEASTS AND DROSOPHILA INTERACT JUST BY CHANCE?

Article Type: SI:Animal Fungus Interactions

Keywords: Coevolution, Chemical Communication, Drosophila, Ehrlichpathway, Fermentation, Interspecific Interaction, Mutualism, Semiochemicals, Yeast

Corresponding Author: Dr. Catrin Sonja Guenther, PhD

Corresponding Author's Institution: University of Lincoln

First Author: Catrin Sonja Guenther, PhD

Order of Authors: Catrin Sonja Guenther, PhD; Matthew R Goddard, Professor

Abstract: The fruit fly Drosophila melanogaster and the baker's yeast Saccharomyces cerevisiae are classic research model organisms that are also associated in nature, at least around vineyards. Sharing the same ephemeral fruit niche, winged Drosophila feed on immotile yeasts. That a yeast diet is essential for larvae development and saprophagous fruit flies are attracted to a suite of yeast volatiles has been well established over the last century. Recently, research has focussed on the potential mutual benefit of this interaction hypothesising yeasts also benefit via dispersal from ephemeral fruits. It now appears the concept of a co-evolved mutualism between yeasts and Drosophila has permeated the literature. However, until robust evidence regarding the evolution and maintenance of this yeast-fly association has been provided, we suggest there is no compelling evidence to reject the more simplistic null hypothesis that these interactions are due to exaptation, and not a mutualism driven by natural selection. 
Commentary

\title{
DO YEASTS AND DROSOPHILA INTERACT JUST BY CHANCE?
}

\author{
Catrin S. Günther**1 and Matthew R. Goddard ${ }^{1,2}$ \\ ${ }^{1}$ School of Life Sciences, University of Lincoln, Joseph Banks Laboratories, Green Lane, \\ Lincoln, LN6 7DL, United Kingdom \\ and \\ ${ }^{2}$ School of Biological Sciences, The University of Auckland, New Zealand \\ *Corresponding author: Catrin S. Günther \\ cgunther@lincoln.ac.uk
}

Tel: 00441522835307 


\begin{abstract}
The fruit fly Drosophila melanogaster and the baker's yeast Saccharomyces cerevisiae are classic research model organisms that are also associated in nature, at least around vineyards. Sharing the same ephemeral fruit niche, winged Drosophila feed on immotile yeasts. That a yeast diet is essential for larvae development, and that saprophagous fruit flies are attracted to a suite of yeast volatiles, has been well established over the last century. Recently, research has focussed on the potential mutual benefit of this interaction hypothesising yeasts also benefit via dispersal from ephemeral fruits. It now appears that the concept of a co-evolved mutualism between yeasts and Drosophila has permeated the literature. However, until robust evidence regarding the evolution and maintenance of this yeast-fly association has been provided, we suggest there is no compelling evidence to reject the more simplistic null hypothesis that these interactions are due to exaptation, and not a mutualism driven by natural selection.
\end{abstract}

\title{
Keywords
}

Coevolution, Chemical Communication, Drosophila, Ehrlich-pathway, Fermentation, Interspecific Interaction, Mutualism, Niche Construction, Semiochemicals, Yeast

\section{INTRODUCTION}

The emergence of Ascomycota, Basidiomycota and Glomales, which comprise the three major groups of fungi, can be dated to 600-million years ago (Mya), and plant-fungus mutualisms are known to have occurred during early colonisation of land by terrestrial plants in the Ordovician about 460 Mya (Redecker et al. 2000), 60-million years before the first 
land-bound insect evolved flight in the Devonian period. Within the Ascomycota, two independent lineages emerged from a metabolically aerobic ancestor about 500 Mya:

1) fission yeasts (Taphrinomycotina)

2) budding yeasts (Saccharomycotina).

Both lineages independently evolved a novel metabolic strategy (the Crabtree effect) where sugars are preferentially fermented even in the presence of oxygen (Dashko et al. 2014). The ability to propagate as facultative anaerobes, however, was modified during further diversification events within the Saccharomycotina subphylum over the last 200 Mya, leading to Crabtree-positive (preferential use of fermentation that can occur simultaneously with respiration) and Crabtree-negative (preferential respiration) species.

Yeasts are often associated with insects and plants (Chandler et al. 2012, Stefanini et al. 2012, Witzgall et al. 2012, Six 2013) and fermenting species quickly dominate in sugar-rich sources such as fruits, where they produce ethanol in the presence of oxygen (preferential fermentation) and other volatile compounds (Cordente et al. 2012). Human history is linked with the utilisation and refinement of food and beverage fermentation by microbes, with evidence of their preparation from rice, honey and fruit (hawthorn and/or grape) as early as 7000 BC (McGovern et al. 2004). Preferential fermentation is one of the most distinguishing features of Saccharomyces species, and this has actively been harnessed by humans to such a degree that certain lineages of this species are termed "domesticated" (Legras et al. 2007, Liti et al. 2009). Drosophila fruit flies of the subgenus Sophophora such as D. melanogaster are human commensals which may also co-inhabit the same ephemeral fruit niche as fermenting yeasts, and they cloud around wineries and often drown themselves in fermented foods, like wine and vinegar. Drosophila evolved in the mid-late Eocene (40 Mya) from a common 
ancestor and further diversified in response to biogeographic events (Izumitani et al. 2016). Drosophila are generally saprophagous microbe-feeders although a trophic shift to herbivory evolved in the subgenus Scaptomyza about 20 Mya which might be linked to the loss or pseudogenisation of odour-receptors crucial for the recognition of yeast volatiles (GoldmanHuertas et al. 2015).

It is well documented that a yeast diet enhances fly fecundity and larvae development (Anagnostou et al. 2010, Rohlfs and Kurschner 2010, Matavelli et al. 2015) and while a few studies provide tentative evidence of a mutualism with specific fly and yeast isolates (Buser et al. 2014, Christiaens et al. 2014), the origin and extent of more general DrosophilaSaccharomyces interactions has not described: are they all mutualisms (see supplementary Table for definition of terms)? Have yeasts and flies generally coevolved as a result of selective pressures to optimise their life history traits and reproductive output? Or has any association arisen by chance from a coincidental combination of pre-existing adaptations (exaptation) of both partners in just a handful of specific situations?

\section{Biochemistry of yeast volatile production and hypotheses for biological benefit}

The evolution of the Crabtree effect allows most Saccharomyces yeasts to employ preferential alcoholic fermentation, even in the presence of oxygen, as powerful means of ecosystem engineering (Goddard 2008, see supplementary Table for definition of terms): in sugar-rich media, glucose is converted to cytotoxic ethanol, carbon dioxide and heat. Although respiration delivers more ATP to the organism, therefore increasing biomass production, preferential fermentation is ecologically successful. Preferential fermentation may act as an antagonistic strategy to both sabotage and outcompete other microorganisms as it allows ATP to be generated more rapidly, which translates to a greater growth rate, and 
simultaneously creates a toxic, hot and alcoholic environment (Goddard 2008; Pfeiffer \& Morley 2014). In addition, along with the predictions from niche-construction theory, Crabtree-positive yeasts are particularly well adapted not only to survive the hostile conditions they create but also to defend carbon resources from competitors as many species have evolved the ability to catabolise ethanol (Thomson et al. 2005; Pfeiffer \& Morley 2014). In addition to ethanol, yeasts also produce short to medium-chain alcohols (fusel alcohols) during assimilation of plant-based amino acids (Fig 1). Fusel alcohols are formed from fusel aldehydes via the Ehrlich pathway and can be further oxidised to organic acids (Hazelwood et al. 2008). These volatile organic compounds may be converted into more complex metabolites by the cell, with one example being alcohol acyl transferases (ATF, Fig 1) which use alcohols as precursors for the formation of esters at the expense of acetyl-CoA or fatty acyl-CoAs that are common intermediates of the primary sugar and fatty acid metabolism. Yeast volatiles may escape the cell and diffuse rapidly through air. Volatile ester biosynthesis and the formation of fusel-like aldehydes, alcohols and acids is not exclusive to yeasts and are common among fruits (El Hadi et al. 2013). However, yeasts alter the composition and concentrations of fruit volatiles to produce a different chemical signature (Cordente et al. 2012). Olfactory recognition of these esters and fusel volatiles is common in vertebrates as well as invertebrates feeding on fruit, which is not surprising considering that these compounds are a signal for food sources. Generally, several biological functions have been hypothesised for volatile ester production in yeasts, summarised by Saerens et al. (2010), and these suggest they act as metabolic 'relief valves', detoxification pathways, or fill an ecological function as infochemicals to promote dispersal by insects. To date, there is no clear evidence that allow these hypotheses to be robustly tested. 
The effects of fungal secondary metabolites on insects can generally be classified into stimulants, attractants, deterrents and repellents (Holighaus \& Rohlfs 2016) which constitute a variety of diverging biological functions. In addition, certain filamentous fungi such as Aspergillus respond to insect-grazing by induced production of mycotoxins, such as the polyketide sterigmatocystin, to successfully defeat their predators (Rohlfs 2015). Although this secondary metabolite is not volatile, polyketides are formed from acetyl-CoA precursors, the same substrate used by ATF for the formation of volatile esters. The enzymatic apparatus required for the biosynthesis of polyketides, however, has not naturally evolved in yeasts. To date, yeast secondary metabolites with insecticidal properties have not been identified whereas there is evidence for their role as insect attractants or repellents, especially in association with Drosophila (Hutner et al. 1937, Becher et al. 2012, Palanca et al. 2013, Scheidler et al. 2015).

\section{The role of yeast volatiles in mediating Drosophila interactions}

That D. melanogaster feasts on yeasts and that this microbial diet is crucial for larval development was first reported by Baumberger (1917) and later confirmed by Phaff (1956) who isolated yeasts from the alimentary canal of fruit flies. It is now established that yeast are an essential staple of Drosophila diet, and common life history traits of D. melanogaster such as survival, development time and adult body weight are influenced by both, yeastspecies and yeast-biomass available to the larvae (Anagnostou et al. 2010). Further, yeast diversity was also shown to positively impact Drosophila life history traits not only by increasing larval development, possibly due to enhanced nutrition, but also by increasing larval survival rates in the presence of antagonistic filamentous fungi (Rohlfs \& Kurschner 2010). Yeasts can detoxify mycotoxins (Hathout \& Aly 2014), and this might add further 
potential benefits for flies. Further, Stamps et al. (2012) observed that D. melanogaster larval activity increased yeast density while simultaneously reducing species diversity to increase yeasts that are more beneficial to larvae. This has been termed 'proto-farming' where growth rate survival and/or density of an edible species (yeast) increases at a locality due to the presence of a "farmer" (larvae).

Given the benefits derived from yeasts, it is unsurprising that D. melanogaster odour receptors respond to yeast volatiles (Hallem and Carlson 2004, 2006). Experiments conducted with a few strains of $S$. cerevisiae, show yeast volatiles can attract fruit flies, stimulate oviposition and affect mating success by increasing sexual receptivity in females (Becher et al. 2012, Gorter et al. 2016). Even in the absence of yeasts, fermentation-like volatiles can lure fruit flies (Stökl et al. 2010) and at least one case of specialised floral mimicry is described in a rainforest orchid which attracts a single drosophilid species (Scaptodrosophila bangi) for pollination (Martos et al. 2015). These examples of chemical mimicry of yeast volatiles by plants suggest a relationship between yeasts and flies that is sufficiently old and stable to have allowed secondary mimics to evolve.

\footnotetext{
Although several volatiles are likely to act as semiochemicals (Table 1, see supplementary Table for definition of terms), the evidence suggests the effectiveness of Drosophila attraction by Saccharomyces yeasts is not controlled by the presence or absence of single compounds but is a function of volatile ratios in combination with a fruit context (Arguello $e t$ al. 2013, Günther et al. 2015). Any yeast-fly interaction has to be considered as part of a tripartite relationship which includes fruit/plant, and this raises the question of to what extent the attractiveness of a particular yeast is contingent on the third plant partner. For example,
} 
yeasts synthesise 3-methylbutanol from L-leucine via the Ehrlich-pathway and the corresponding acetate ester is known to mediate $D$. simulans attraction, but only when presented in a fruit context (Günther et al. 2015).

At first inspection the nature of the yeast-Drosophila association appears to be one of predation, i.e. it comprises benefit only for Drosophila. Usually organisms avoid predation, be it through defence (chemical or physical) or escape. Ethanol production by yeasts might have acted as a predator repellent ancestrally, but it cannot be regarded as an anti-predator adaptation against fruit flies today. Yeasts share the same ephemeral fruit niche with insects such as Drosophila. However, one key difference between these species is that yeasts are immotile and depend on dispersal via other agents to persist. Following this, one idea suggests that yeast volatile production is a means of chemical communication (see supplementary Table for definition of terms) with microbe-feeding flies, and this is beneficial as it increases the propensity of yeast dispersal to new habitats by flies (Saerens et al. 2010, Buser et al. 2014, Christiaens et al. 2014). If this were the case then the yeast-fly association would be bidirectional and resemble characters of mutualism, where both partners must receive increased fitness from the association, and not a simple predator-prey interaction where only one side of the association realises fitness increases (West et al. 2007). If this proposed mutualism was maintained by chemical communication then particular yeast volatiles will be under selection, and research suggests that volatile ester production, especially the acetate esters ethyl acetate and 3-methylbutyl acetate (Christiaens et al. 2014, Günther et al. 2015), is important for Drosophila attraction.

\section{Are yeast-fly associations just chance?}


The mutualism hypothesis articulated above appears intuitively attractive, but the evidence to support this is weak and sporadic. D. melanogaster and S. cerevisiae are significant model organisms in their own right and thus very good candidates to study interspecific interactions of microbes and insects. D. melanogaster is likely the best studied animal model which has been used for genetic research for over a century and S. cerevisiae was the first eukaryote to have its genome sequenced in 1996. Despite this, the ecological reality of these organisms is embedded in multitrophic networks, and any data generated from their analyses in isolation may not be representative:

First, correlations between attraction and yeast dispersal have only been shown for three $S$. cerevisiae genotypes with just two Drosophila isolates (one $D$. simulans and one $D$. melanogaster, Buser et al. 2014, Christiaens et al. 2014). Further, some S. cerevisiae genotypes are repulsive, and others have different levels of attractiveness, to at least two isofemale lines of Drosophila (one D. simulans and one D. melanogaster; Buser et al. 2014; Palanca et al. 2013), demonstrating that attraction is by no means a fixed trait. In addition, $S$. cerevisiae, is not abundant on fruit and rarely associated with D. melanogaster naturally (Goddard \& Greig, 2015, Hoang et al. 2015, Lam \& Howell 2015). Together this calls an evolved mutualism of these species into question. Other than S. cerevisiae, olfactory response of D. melanogaster was evaluated to a limited range of Saccharomycetales yeasts from other genera, such as Hanseniaspora uvarum and Pichia kluyverii (Palanca et al 2013, Hoang et al. 2015, Scheidler et al 2015), which are more commonly associated with Drosophila in nature (Dobzhanski et al. 1956, Hamby et al. 2012, Lam \& Howell 2015). However, whether attraction corresponds with success in yeast dispersal for these is not known. 
Second, one might be tempted to view a mutualism between yeasts and flies as underpinned by adaptation of the expression of atfl for acetate ester formation in yeasts and the corresponding odour receptor genes (or43b, or $47 a$, or $85 b)$ in Drosophila. Chemical signals can evolve from cues (Steiger et al. 2011, see supplementary Table for definition of terms) but to date there are no compelling data to suggest that yeast volatile production has evolved to establish a mutualism. Further, in evolutionary terms, budding yeasts are hundreds of millions of years older than Drosophila fruit flies and we have little data concerning the history of the interaction between these two organisms. Despite their long-standing status as model organisms, genetic evidence of traits selected for as a result of their interactions in nature is scarce. Although several studies imply the coevolution of yeasts and flies (Stökl et al. 2010, Goldman-Huertas et al. 2015, Martos et al. 2015) mechanisms supporting their molecular evolution are yet to be elucidated. Odour receptors responding to yeast volatiles such as acetate esters are generally broadly tuned (Mansourian \& Stensmyr 2015) conferring attraction to a range of chemically similar compounds. Therefore, volatiles that stimulate antennal responses and might influence behaviour are not strictly yeast-specific. Adaptive evolution has been described for the alcohol dehydrogenase (Adh) locus in Drosophila which is crucial for the detoxification and carbon-recycling of ethanol and at least three independent parallel-evolutionary events have resulted in novel protein functions of the ancestral gene between 2-30 Mya (Jones \& Begun 2005). Ethanol tolerance is linked to ADH protein levels in Drosophila and differs drastically even between evolutionary sibling species such as $D$. melanogaster and D. simulans with the latter showing reduced ethanol tolerance and lower ADH-levels (Laurie et al. 1990). Ethanol adaptation has also been linked to acetic acid tolerance in D. melanogaster and was suggested as a mechanism to reduce environmental ethanol stress and allow colonisation of substrates altered through microbial infestation (Chakir et al. 1993). While differential adaptations to ethanol and acetic acid are likely to 
impact yeast preference and D. simulans was repulsed by acetic acid (Günther et al. 2015), both sympatric Drosophila species are attracted to the Brewer's yeast S. cerevisiae.

Third, the ecological relationship of Saccharomyces and Drosophila is far from exclusive. Saccharomyces are not only associated with fruit flies, but other insects including bees and wasps (Goddard et al. 2010; Stefanini et al. 2012). While specific flies prefer particular yeasts over others (Palanca et al. 2013, Buser et al. 2014, Scheidler et al. 2015) and differ in their attraction to infested substrates (Matavelli et al. 2015, Date et al. 2017), only a few genera of yeasts are consistently associated with fruit fly populations. Frequently isolated yeasts include Candida, Pichia, Hanseniaspora, Metschnikowia, Torulaspora but rarely Saccharomyces (Hamby et al. 2012, Stamps et al. 2012, Buser et al. 2014, Lam and Howell 2015). None of these yeasts are exclusively associated with Drosophila, and distributions of these yeasts have been shown to be more strongly influenced by Drosophila diet rather than fly species in at least fifteen common Drosophila populations (Chandler et al. 2012). Although flies might discriminate for some yeasts, alternative species are likely to compensate when the favourite food is absent (Dobzhansky et al. 1956). Further, it has long been known that flies are not only associated with yeasts but also bacteria (Baumberger 1917). The bacterial gut commensals Lactobacillus and Acetobacter are indirectly involved in yeast-fly associations by enhancing the flies' appetite for dietary yeasts in response to amino acid deprivation (Leitao-Goncalves et al. 2017). Although bacterial volatiles generally trigger a different set of odour receptors in the fly, Drosophila is also attracted to volatile amines released during bacterial decomposition of organic matter (Min et al. 2013). Throughout the literature a subset of volatiles (Table 1) has been suggested to act as semiochemicals, mediating fruit fly attraction. These volatiles are not only produced by a range of yeasts but also by bacteria and some fruits: 
Small quantities of acetic acid are released during fermentation by yeasts, but the accumulation of acetic acid is more commonly a result of oxidative fermentation of ethanol by acetic acid bacteria. Although ambiguous in its role as fruit fly attractant, acetic acid can be a very effective lure for the vinegar fly $D$. melanogaster, enhance mating success and stimulate oviposition (Joseph et al. 2009, Cha et al. 2012, Gorter et al. 2016). Other key volatiles for Drosophila attraction, namely 3-methyl butanol and acetoin also comprise major volatiles produced by the human skin microbiota, for example by Staphylococcus epidermidis (Verhulst et al. 2009). Therefore, Drosophila attraction to a particular substrate is likely the result of microbial activity, involving yeast and/or bacteria and both microbes are potentially dispersed by the fly. Lastly, O'Conner et al. (2014) hypothesised that the adaptive radiation of Drosophila populations endemic to Hawaii was linked to plant colonisation with symbiotic yeasts, and that flies might be radiating with the microbes rather than host plants themselves. Recent studies testing host plant specificity of cactophilic D. majovensis state that olfactory preference for the plant species can shift in response to plant-microbe and microbe-microbe interactions thus emphasising the importance of each partner to volatile composition in this three-way interaction (Date et al. 2017). While yeasts might play a role in Drosophila phylogenetic diversification, there are no data to suggest that yeast speciation coevolved with Drosophila or flying insects in general, as yeasts evolved before flying insects emerged.

\begin{abstract}
Although a few studies have provided evidence that interactions between specific Drosophila and $S$. cerevisiae isolates may be classed as a facultative mutualism, there is no evidence to support the claim that this may be generalised further. At present there is no compelling evidence to reject the null hypothesis that general interactions between Drosophila and Saccharomyces are either coincidental, fortuitously resulting from pre-existing traits, i.e. this interaction is an exaptation. At most the evidence supports a one-way interaction in terms of
\end{abstract}


benefits for Drosophila. If yeast volatile production were an adaptation acting to attract insects, then this would have to be classed as a generalist approach, targeting a broad range of flying vectors (Table 1) including wasps, moths and beetles in addition to Drosophila. Taking a generalist approach in enhancing odours to attract a variety of vectors rather than relying on one species for dispersal seems a strategy that selection would more readily operate on, but is a more elusive idea to test, and there is no evidence that flying insects other than Drosophila derive fitness benefits from their association with yeasts.

It is desirable to consider experiments that could rigorously test hypotheses regarding the nature of interactions between yeast and flies. The facultative nature of these associations makes such experiments hard to conduct. Ultimately these would need to assay the fitness benefits and costs of specific associations. Buser et al. (2014) have done this in a limited way and found different extents of yeast fitness (measured by dispersal) among isolates. First one would need to assay multiple combinations of fly and yeast genotypes to evaluate the space over which both fly and yeast fitness were increased: if this were large, a more general claim for a mutualistic interaction would be supported. This is a huge task. Another approach is to evaluate the degree to which these interaction and fitness benefit traits are malleable. If these traits change readily over a few generations, this suggests that such associations are not acted on strongly by selection: i.e. they are ephemeral, just like the fruit that yeasts and Drosophila temporarily co-inhabit.

\section{ACKNOWLEDGEMENTS}

We would like to thank Dr Sandra Varga for sharing her views on evolutionary fungal ecology and for critical comments on the manuscript. 


\section{REFERENCES}

Anagnostou C, Dorsch M and Rohlfs M, 2010. Influence of dietary yeasts on Drosophila melanogaster life-history traits. Entomologia Experimentalis Et Applicata 136:1-11.

Arguello JR, Sellanes C, Lou YR, and Raguso RA, 2013. Can Yeast (S. cerevisiae) Metabolic Volatiles Provide Polymorphic Signaling? Plos One 8: e70219

Baumberger JP, 1917. The food of Drosophila melanogaster meigen. Proceedings of the National Academy of Sciences of the United States of America 3:122-126.

Becher PG, Flick G, Rozpedowska E, Schmidt A, Hagman A, Lebreton S, M C. Larsson MC, Hansson BS, Piskur J, Witzgall P, and Bengtsson M, 2012. Yeast, not fruit volatiles mediate Drosophila melanogaster attraction, oviposition and development. Functional Ecology 26:822-828.

Brand JM, Schultz J, Barras SJ, Edson LJ, Payne TL, and Hedden RL, 1977. Bark-Beetle Pheromones - Enhancement of Dendroctonus Frontalis (Coleoptera-Scolytidae) Aggregation Pheromone by Yeast Metabolites in Laboratory Bioassays. Journal of Chemical Ecology 3:657-666

Buser CC, Newcomb RD, Gaskett AC, and Goddard MR, 2014. Niche construction initiates the evolution of mutualistic interactions. Ecology Letters 17:1257-1264.

Cha D H, Adams T, Rogg H, and Landolt PJ, 2012. Identification and field evaluation of fermentation volatiles from wine and vinegar that mediate attraction of spotted wing Drosophila, Drosophila suzukii. Journal of Chemical Ecology 38:1419-1431.

Chakir M, Peridy O, Capy P, Pla E, and David JR, 1993. Adaptation to Alcoholic Fermentation in Drosophila - a Parallel Selection Imposed by Environmental Ethanol and Acetic-Acid. Proceedings of the National Academy of Sciences of the United States of America 90:3621-3625. 
Chandler, JA, Eisen JA, and Kopp A, 2012. Yeast Communities of Diverse Drosophila Species: Comparison of Two Symbiont Groups in the Same Hosts. Applied and Environmental Microbiology 78:7327-7336.

Christiaens JF, Franco LM, Cools TL, De Meester L, Michiels J, Wenseleers T, Hassan BA, Yaksi E, and Verstrepen KJ, 2014. The Fungal Aroma Gene ATF1 Promotes Dispersal of Yeast Cells through Insect Vectors. Cell Reports 9:425-432.

Cordente AG, Curtin CD, Varela C, and Pretorius IS, 2012. Flavour-active wine yeasts. Applied Microbiology and Biotechnology 96:601-618.

Dashko S, Zhou N, Compagno C, and Piskur J, 2014. Why, when, and how did yeast evolve alcoholic fermentation? FEMS Yeast Research 14:826-832.

Date P, Crowley-Gall A, Diefendorf AF, and Rollmann SM, 2017. Population differences in host plant preference and the importance of yeast and plant substrate to volatile composition. Ecology and Evolution 7:3815-3825.

Davis TS, Boundy-Mills K, and Landolt PJ, 2012. Volatile Emissions from an Epiphytic Fungus are Semiochemicals for Eusocial Wasps. Microbial Ecology 64:1056-1063.

Davis TS, and Landolt PJ, 2013. A Survey of Insect Assemblages Responding to Volatiles from a Ubiquitous Fungus in an Agricultural Landscape. Journal of Chemical Ecology 39:860-868.

El-Sayed AM, Heppelthwaite VJ, Manning LM, Gibb AR, and Suckling DM, 2005. Volatile constituents of fermented sugar baits and their attraction to Lepidopteran species. Journal of Agricultural and Food Chemistry 53:953-958.

El Hadi MAM, Zhang FJ, Wu FF, Zhou CH, and Tao J, 2013. Advances in Fruit Aroma Volatile Research. Molecules 18:8200-8229.

Goddard MR, 2008. Quantifying the complexities of Saccharomyces cerevisiae's ecosystem engineering via fermentation. Ecology 89:2077-2082. 
Goddard MR, Anfang N, Tang R, Gardner RC, Jun C, 2010 A distinct population of Saccharomyces cerevisiae in New Zealand: evidence for local dispersal by insects and human-aided global dispersal in oak barrels. Environmental Microbiology 12:63-73

Goddard M. R., and Greig D, 2015. Saccharomyces cerevisiae: a nomadic yeast with niche? FEMS Yeast Research 15 (3).

Goldman-Huertas B, Mitchell RF, Lapoint RF, Faucher CP, Hildebrand JG, and Whiteman NK, 2015. Evolution of herbivory in Drosophilidae linked to loss of behaviors, antennal responses, odorant receptors, and ancestral diet. Proceedings of the National Academy of Sciences of the United States of America 112:3026-3031.

Gorter JA, Jagadeesh S, Gahr C, Boonekamp JJ, Levine JD, and Billeter JC, 2016. The nutritional and hedonic value of food modulate sexual receptivity in Drosophila melanogaster females. Scientific Reports 6:19441

Günther CS, Goddard MR, Newcomb RD, and Buser CC, 2015. The Context of Chemical Communication Driving a Mutualism. Journal of Chemical Ecology 41:929-936.

Phaff HJ, Miller MW, Recca JA, Shifrine M and Mrak EM, 1956. Yeasts Found in the Alimentary Canal of Drosophila. Ecology and Evolution 37:533-538.

Hallem EA, and Carlson JR, 2004. The odor coding system of Drosophila. Trends in Genetics 20:453-459.

Hallem EA, and Carlson JR, 2006. Coding of odors by a receptor repertoire. Cell 125:143160.

Hamby KA, Hernandez A, Boundy-Mills K, and Zalom FG, 2012. Associations of Yeasts with Spotted-Wing Drosophila (Drosophila suzukii; Diptera: Drosophilidae) in Cherries and Raspberries. Applied and Environmental Microbiology 78:4869-4873.

Hathout SH, Aly SE, 2014. Biological detoxification of mycotoxins: a review. Annals of Microbiology 64: 905-919 
Hazelwood LA, Daran JM, van Maris AJA, Pronk JT, and Dickinson JR, 2008. The Ehrlich pathway for fusel alcohol production: a century of research on Saccharomyces cerevisiae metabolism. Applied and Environmental Microbiology 74:3920-3920.

Hoang D, Kopp A, and Chandler JA, 2015. Interactions between Drosophila and its natural yeast symbionts-Is Saccharomyces cerevisiae a good model for studying the fly-yeast relationship? PeerJ 3:e1116

Holighaus G, and Rohlfs M, 2016. Fungal allelochemicals in insect pest management. Applied Microbiology and Biotechnology 100:5681-5689.

Hutner SH, Kaplan HM, and Enzmann EV, 1937. Chemicals attracting Drosophila. The American Naturalist 71:575-581.

Izumitani HF, Kusaka Y, Koshikawa S, Toda MJ, and Katoh T, 2016. Phylogeography of the Subgenus Drosophila (Diptera: Drosophilidae): Evolutionary History of Faunal Divergence between the Old and the New Worlds. Plos One 11: e0160051

Jones CD, and Begun DJ, 2005. Parallel evolution of chimeric fusion genes. Proceedings of the National Academy of Sciences of the United States of America 102:11373-11378.

Joseph, RM, Devineni AV, King IFG, and Heberlein U, 2009. Oviposition preference for and positional avoidance of acetic acid provide a model for competing behavioral drives in Drosophila. Proceedings of the National Academy of Sciences of the United States of America 106:11352-11357.

Lam SSTH, and K. S. Howell K,. 2015. Drosophila-associated yeast species in vineyard ecosystems. FEMS Microbiology Letters 362:fnv170

Landolt PJ, Toth M, Meagher RL, and Szarukan I, 2013. Interaction of acetic acid and phenylacetaldehyde as attractants for trapping pest species of moths (Lepidoptera: Noctuidae). Pest Management Science 69:245-249. 
Laurie CC, Heath EM, Jacobson JW, and Thomson MS, 1990. Genetic-Basis of the Difference in Alcohol Dehydrogenase Expression between Drosophila melanogaster and Drosophila simulans. Proceedings of the National Academy of Sciences of the United States of America 87:9674-9678.

Legras JL, Merdinoglu D, Cornuet JM, and Karst F, 2007. Bread, beer and wine: Saccharomyces cerevisiae diversity reflects human history. Molecular Ecology 16:2091-2102.

Leitao-Goncalves R, Carvalho-Santos Z, Francisco AP, Fioreze GT, Anjos M, Baltazar C, Elias AP, Itskov PM, Piper MDW, and Ribeiro C, 2017. Commensal bacteria and essential amino acids control food choice behavior and reproduction. Plos Biology 15: e2000862.

Liti G, Carter DM, Moses AM, Warringer J, Parts L, James SA, Davey RP, Roberts IN, Burt A, Koufopanou V, Tsai IJ, Bergman CM, Bensasson D, O'Kelly MJT, van Oudenaarden A, Barton D BH, Bailes E, Ba ANN, Jones M, Quail MA, Goodhead I, Sims S, Smith F, Blomberg A, Durbin R, and Louis EJ, 2009. Population genomics of domestic and wild yeasts. Nature 458:337-341.

Mansourian S, and Stensmyr MC, 2015. The chemical ecology of the fly. Current Opinion in Neurobiology 34:95-102.

Martos F, Cariou ML, Pailler T, Fournel J, Bytebier B, and Johnson SD, 2015. Chemical and morphological filters in a specialized floral mimicry system. New Phytologist 207:225-234.

Matavelli C, Carvalho MJA, Martins NE, and Mirth CK, 2015. Differences in larval nutritional requirements and female oviposition preference reflect the order of fruit colonization of Zaprionus indianus and Drosophila simulans. Journal of Insect Physiology 82:66-74. 
McGovern PE, Zhang JH, Tang JG, Zhang ZQ, Hall GR, Moreau RA, Nunez A, Butrym ED, Richards MP, Wang CS, Cheng GS, Zhao ZJ, and Wang CS, 2004. Fermented beverages of pre- and proto-historic China. Proceedings of the National Academy of Sciences of the United States of America 101:17593-17598.

Min S, Ai M, Shin SA, Shu GS, 2013. Dedicated olfactory neurons mediating attraction behaviour to ammonia and amines in Drosophila. Proceedings of the National Academy of Sciences of the United States of America 110:E1321-E1329.

Nout MJR, and Bartelt RJ, 1998. Attraction of a flying nitidulid (Carpophilus humeralis) to volatiles produced by yeasts grown on sweet corn and a corn-based medium. Journal of Chemical Ecology 24:1217-1239.

O'Connor TK, Humphrey PT, Lapoint RT, Whiteman NK, and O'Grady PM, 2014. Microbial Interactions and the Ecology and Evolution of Hawaiian Drosophilidae. Frontiers in Microbiology 5:616

Palanca L, Gaskett AC, Günther CS, Newcomb RD, and Goddard M. R, 2013. Quantifying Variation in the Ability of Yeasts to Attract Drosophila melanogaster. Plos One 8:e75332

Phelan PL, and HC Lin, 1991. Chemical Characterization of Fruit and Fungal Volatiles Attractive to Dried-Fruit Beetle, Carpophilus hemipterus (L) (Coleoptera, Nitidulidae). Journal of Chemical Ecology 17:1253-1272.

Pfeiffer T, and Morley A, 2014. An evolutionary perspective on the Crabtree effect. Frontiers in Molecular Biosciences 1: 1-6

Redecker D, Kodner R, and Graham LE, 2000. Glomalean fungi from the Ordovician. Science 289:1920-1921.

Rohlfs M, 2015. Fungal secondary metabolite dynamics in fungus-grazer interactions: novel insights and unanswered questions. Frontiers in Microbiology 5:Article 788 
Rohlfs M, and Kurschner L, 2010. Saprophagous insect larvae, Drosophila melanogaster, profit from increased species richness in beneficial microbes. Journal of Applied Entomology 134:667-671.

Saerens SMG, Delvaux FR, Verstrepen KJ, Thevelein JM, 2010. Production and biological function of volatile esters in Saccharomyces cerevisiae. Microbial Biotechnology 3:165-177.

Scheidler NH, Liu C, Hamby KA, Zalom FG, and Syed Z, 2015. Volatile codes: Correlation of olfactory signals and reception in Drosophila-yeast chemical communication. Scientific Reports 5:Article 14059

Six DL, 2013. The Bark Beetle Holobiont: Why Microbes Matter. Journal of Chemical Ecology 39:989-1002.

Stamps JA, Yang LH, Morales VM, and Boundy-Mills KL, 2012. Drosophila Regulate Yeast Density and Increase Yeast Community Similarity in a Natural Substrate. Plos One 7:e42238

Stefanini I, Dapporto L, Legras JL, Calabretta A, Di Paola M, De Filippo C, Viola R, Capretti P, Polsinelli M, Turillazzi S, and Cavalieri D, 2012. Role of social wasps in Saccharomyces cerevisiae ecology and evolution. Proceedings of the National Academy of Sciences of the United States of America 109:13398-13403.

Steiger S, Schmitt T, and Schaefer HM, 2011. The origin and dynamic evolution of chemical information transfer. Proceedings of the Royal Society B-Biological Sciences 278:970-979.

Stökl J, Strutz A, Dafni A, Svatos A, Doubsky J, Knaden M, Sachse S, Hansson BS, and Stensmyr MC, 2010. A Deceptive Pollination System Targeting Drosophilids through Olfactory Mimicry of Yeast. Current Biology 20:1846-1852. 
Dobzhansky T, Cooper DM, Phaff HJ, Knapp EP, and Carson HL, 1956. Differential Attraction of Species of Drosophila to Different Species of Yeasts. Ecology 37:544550.

Thomson JM, Gaucher EA, Burgan MF, De Kee DW, Li T, Aris JP, and Benner SA, 2005. Resurrecting ancestral alcohol dehydrogenases from yeast. Nature Genetics 37:630635.

Verhulst NO, Beijleveld H, Knols BGJ, Takken W, Schraa G, Bouwmeester HJ, and Smallegange R C, 2009. Cultured skin microbiota attracts malaria mosquitoes. Malaria Journal 8:302

West SA, Griffin AS, and Gardner A, 2007. Social semantics: altruism, cooperation, mutualism, strong reciprocity and group selection. Journal of Evolutionary Biology 20:415-432.

Witzgall P, Proffit M, Rozpedowska E, Becher PG, Andreadis S, Coracini M, Lindblom TUT, Ream LJ, Hagman A, Bengtsson M, Kurtzman CP, Piskur J, and Knight A, 2012. "This is not an Apple"-Yeast Mutualism in Codling Moth. Journal of Chemical Ecology 38:949-957.

\section{CAPTIONS}

Figure 1: Volatile production in yeast. Linking Ehrlich-pathway with fatty acid biosynthesis and preferential fermentation (in the presence of oxygen). ATF: Alcohol acyl transferase; CA: Acetyl-CoA carboxylase 


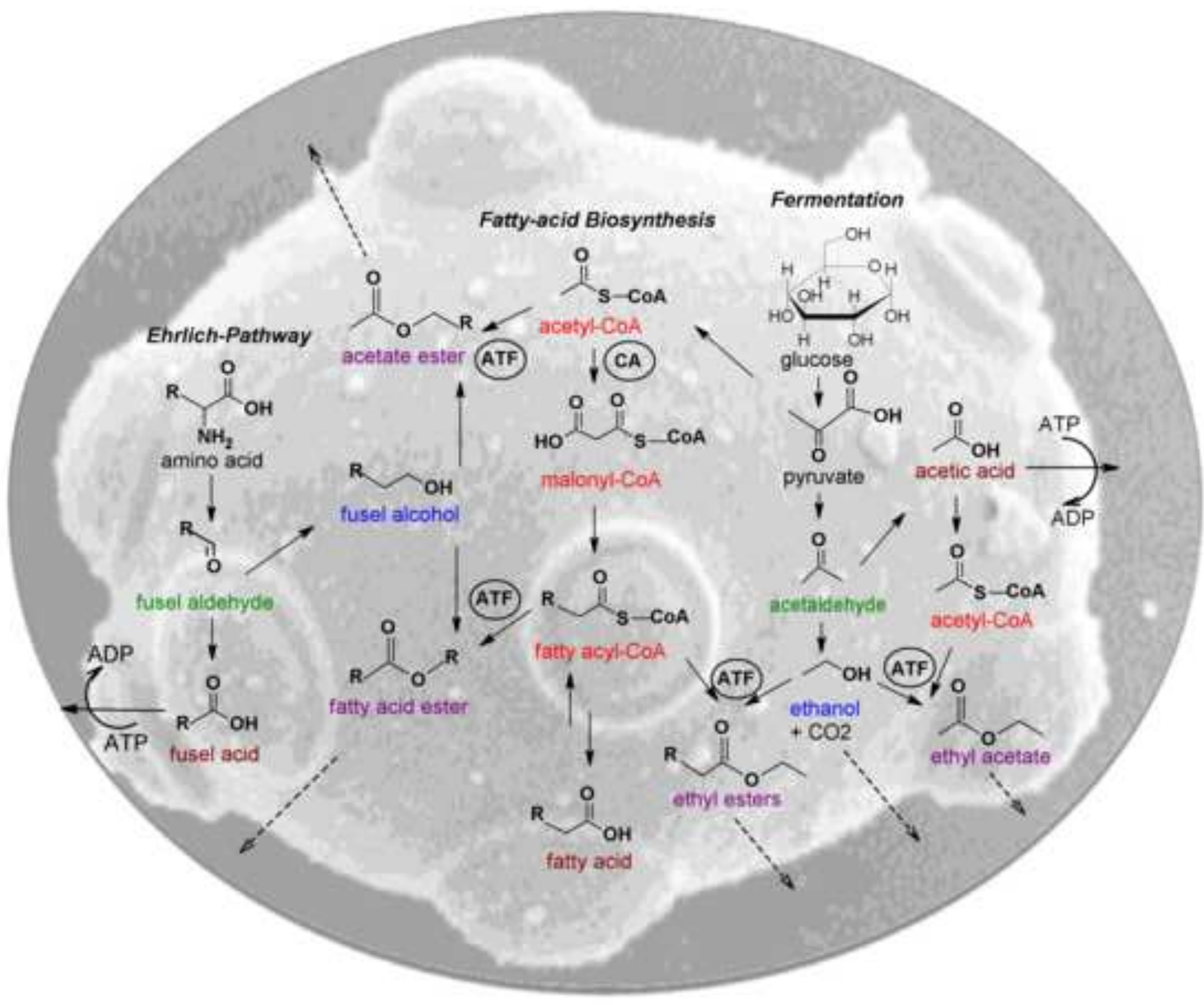


Table 1: Yeast volatiles and their potential role as semiochemicals for insect attraction.

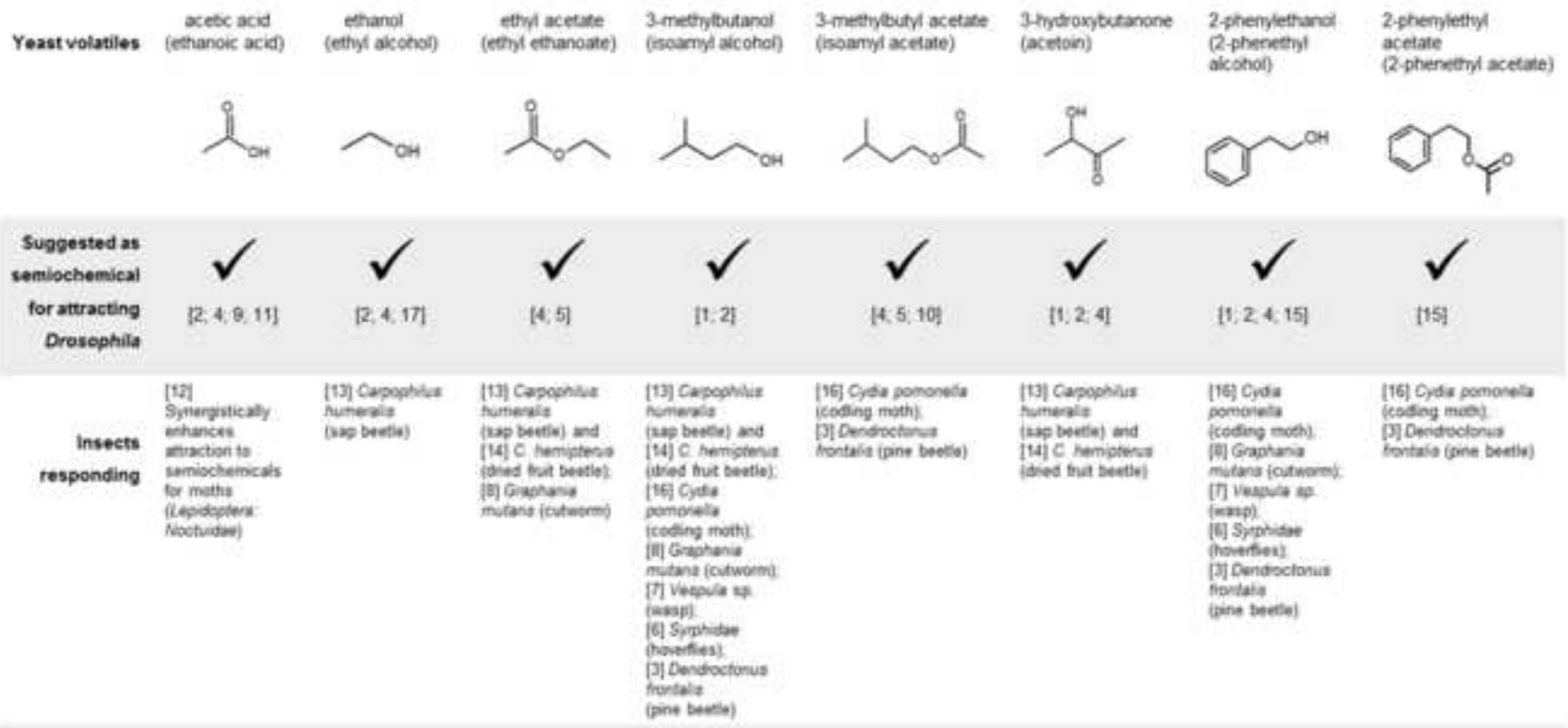

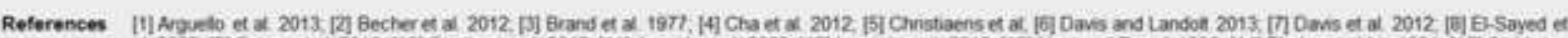

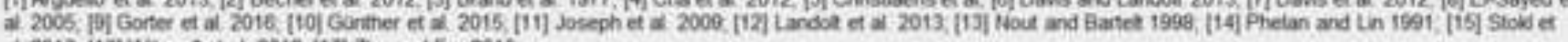

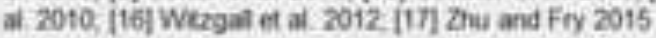


e-component
Click here to download e-component: supplementary Table_FUNECO-D-17-00277.docx e-component
Click here to o (a)

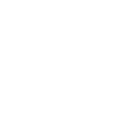
(n)

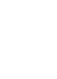

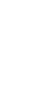

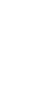

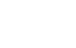
(1)

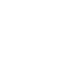

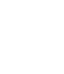

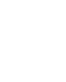

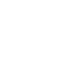

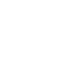

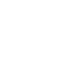

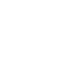

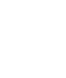

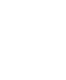

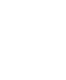
. . 